Artículos 



\title{
Escepticismo, mecanicismo, teología y alquimia en Robert Boyle*
}

\section{Carmen Silva}

\begin{abstract}
Debemos intentar aproximarnos a la verdad, aún cuando no la aprehendamos completamente, así podremos estar más cerca de lo que estamos ahora.
\end{abstract}

Galeno $^{1}$

\section{I} ntroducción

Nuestro objetivo es aproximarnos a la naturaleza del mecanicismo de Robert Boyle y, de esta forma, poder apreciar cómo esta tesis o modelo explicativo que fue fundamental para los filósofos naturales del siglo XVII, tuvo variantes importantes entre sus defensores.

Me parece que en Boyle encontramos ciertos rasgos que definen $s u$ mecanicismo y, por tanto, su filosofía natural; algunos de ellos los comparte con contemporáneos suyos, y otros no, pues en este periodo encontramos que los autores se enfrentaron de forma diferente al escepticismo pirrónico, lo que dio como resultado una epistemología específica de la que surgió en parte la filosofía natural, la cual a su vez estaba estrechamente vinculada a una determinada perspectiva teológica. En otras palabras, que las diferentes combinaciones de las diversas posturas, tanto teológicas como epistemológicas, dieron lugar a una determinada filosofía natural.

En resumen, en este trabajo pretendo hacer un bosquejo de todo esto exponiendo algunos de los elementos epistémico y teológicos que componen la filosofía natural de Robert Boyle y, por tanto, aproximarnos a la naturaleza de su mecanicismo.

* Una versión previa de este trabajo fue leída en el XII Congreso Nacional de Filosofía en Neuquen, Argentina, en diciembre del 2003. Otra versión resumida del mismo fue enviada en abril de 2004 para su publicación en las memorias de dicho Congreso. Agradezco a José Antonio Robles y Alejandra Velásquez su lectura y comentarios a las diferentes versiones de este trabajo.

${ }^{1}$ Este epígrafe lo pone Boyle en su libro A Free Enquiry into the Vulgarly Received Notion of Nature. Cambridge, Nueva York, Universidad de Cambridge, 1996. 
Antes de cerrar la introducción comentaré en forma breve, que la filosofía natural de Robert Boyle es una continuación de ciertas posturas de la filosofía de Gassendi, por ejemplo: 1) el escepticismo constructivo, 2) su mecanicismo, pues el mecanicismo que impactó a la mayoría de los pensadores ingleses de su época, fue principalmente el de Gassendi, gracias a los trabajos de difusión de la obra gassendiana por medio de Walter Charleton, 3) igualmente, la posición teológica de Roberte Boyle, a saber, el voluntarismo teológico es la misma que la de Gassendi, pues ésta permite reconciliar el mecanicismo con el cristianismo y también el escepticismo con la filosofía natural. Además existen otros dos aspectos importantes de la filosofía natural de Robert Boyle que no son una continuación directa de Gassendi: 1. El desarrollo de la experimentación que tiene como antecedente directo a Francis Bacon. 2. La alquimia que es una continuidad renovada del hermetismo del Renacimiento, teniendo como antecedente a Paracelso, la cual cumple varias funciones, a saber: cristianizar el atomismo, rebasar los límites de la explicación mecánica y, por último, apoyar una reforma de la medicina.

Para cerrar la introducción, cito a continuación un pasaje de Margaret Osler que me parece muy sugerente: "En lugar de ver a la historia del pensamiento moderno temprano, simplemente como un triunfo de los modernos, puede comprenderse por lo menos en parte, como la sustitución de un modelo antiguo por otro". ${ }^{2}$

\section{Escepticismo}

El escepticismo en el siglo XVII

Richad Popkin, el especialista en historia del escepticismo, afirma que una vez que el escepticismo pirrónico llegó al Renacimiento, fortaleció a la Reforma luterana sobre el problema del criterio y pasó a los modernos generando dos alternativas o dos respuestas frente al mismo, a saber: “...el nuevo racionalismo de René Descartes y 'el escepticismo constructivo' de Petrus Gassendi y Marin Mersenne". ${ }^{3}$

2 Margaret Osler, ed., "Presentación", en Atoms, Pneuma and Tranquility. Epicurean and Stoic Themes in European Thought. Cambridge, Universidad de Cambridge, 1991.

${ }^{3}$ Richard Popkin, La historia del escepticismo desde Erasmo a Spinoza. México, FCE, 1983 , p. 45. 
En otras palabras, al término del siglo XVI y durante el XVII existieron dos rutas o dos formas de enfrentar el escepticismo pirrónico; la primera es la que mantenía que era posible superarlo, defendiendo un conocimiento que se identificaba con la certeza; esta vía pretendió superar el escepticismo teniendo como modelo de conocimiento a la matemática, la cual es un tipo de conocimiento que sí puede superar las dudas del escéptico según sus defensores; también porque afirmaba acceder al conocimiento de las esencias. La garantía de este conocimiento está en su método para guiar correctamente a la razón, en la metafísica y en los fundamentos y criterios epistémicos que son objeto de la mente humana. Esta vía es la propia de Descartes y sus seguidores.

La segunda vía, como lo afirma Popkin en el pasaje antes citado, es la propuesta por Gassendi y Merssene, que es la del escepticismo académico, o mitigado, que sostiene que la única forma de conocimiento asequible para el hombre, es el conocimiento probable, pues según esta ruta epistemológica el conocimiento de las esencias es imposible; lo que es posible es el conocimiento de las apariencias o fenómenos; por tanto, el fundamento del conocimiento no está en la razón sino en la experiencia, y el criterio y método para no errar, ni caer en el dogmatismo, es no rebasar los límites del entendimiento humano. Esta epistemología, que será el fundamento de cierta filosofía natural, tiene entre sus representantes más notables a Pierre Gassendi, Marin Mersenne, Francis Bacon, Robert Boyle, John Locke, Berkeley, Newton y a la misma Royal Society.

Me parece que estas dos vías epistémicas, resultado del enfrentamiento con el escepticismo pirrónico, son el fundamento de dos propuestas en filosofía natural, alternativas al modelo aristotélico-tomista y que, a partir de ellas, podemos hablar de dos formas de mecanicismo en el siglo XVII, es decir, uno apoyado en la epistemología de corte cartesiano y, el otro, compatible con una epistemología probabilista y empirista. ${ }^{4}$

Koyré, en el siguiente pasaje sobre Gassendi, avala mi sugerencia de las dos epistemologías: “...este encarnizado adversario de Aristóteles, este decidido partidario de Galileo permanece ajeno al espíritu de la ciencia moderna, y especialmente al espíritu de matematización que la anima". ${ }^{5}$

${ }^{4}$ En un trabajo anterior mío titulado "Origen y naturaleza del mecanicismo en el siglo XVII”, en L. Benítez, Z. Monroy y J. A. Robles, coords, La filosofía natural y la filosofía moral en la modernidad. México, UNAM, 2003, llamé al primero mecanicismo matemático o real y al segundo empírico o fenoménico.

${ }^{5}$ Alexandre Koyré, Estudios de historia del pensamiento científico. México, Siglo XXI, 1977, p. 306. Igualmente, sobre la distinción entre matemático y empírico podemos encontrar otra distinción dentro de la matemática, que me sugirió Leonel Toledo a saber: 1. La matemática platónica que siguieron autores como Cusa y Bruno, que es de carácter teológico-ontológico y 2. La de Arquímedes que es más práctica y, 
Quizá Newton es el filósofo natural más importante dentro de lo que Dijksterhuis llama la mecánica clásica, pues él representa una síntesis de estos dos modelos mecánicos, a saber: el matemático y el empírico.

Pasemos ahora al tema del escepticismo en Robert Boyle para poder apreciar las peculiaridades de $s u$ mecanicismo.

\section{La epistemología de Robert Boyle}

Por lo que decíamos líneas arriba, Boyle es seguidor de la ruta propuesta por Gassendi y Mersenne frente al escepticismo pirrónico; a esta ruta Popkin le llama escepticismo constructivo. Las características que conforma la epistemología boyleana relacionada con este tipo de escepticismo se expresan en los siguientes términos: certeza moral, demostración moral, y finalmente algo muy nuevo y que Boyle aporta a la nueva filosofía natural, a saber, la experimentación como un medio de encontrar y corroborar conocimientos. Otro elemento notable y peculiar de este autor de origen irlandés y que en gran medida representa una actitud epistemológica extendida en ese periodo en la filosofía de lengua inglesa, es el uso del lenguaje y procedimientos jurídicos en la filosofía natural y especulativa. Sobre este tema Popkin dice lo siguiente: "La teoría del escepticismo mitigado y limitado desarrollada por los teólogos anglicanos se convirtió en la teoría de la Royal Society y también de las Cortes inglesas". ${ }^{6}$

La certeza moral se encuentra situada entre el conocimiento absolutamente cierto y la mera opinión. Como vemos, en el pensamiento moderno a la par que se pretendía postular una nueva filosofía natural, este objetivo obligaba a redefinir una teoría del conocimiento. ${ }^{7}$

Otra perspectiva epistemológica interesante es la aceptación o concepción del conocimiento como algo corregible, lo cual está relacionado, por un lado, al conocimiento experimental o empírico que siempre es corregible y, por el otro, a una perspectiva teológica particular y, desde luego, ambas están ligadas a una postura frente al escepticismo pirrónico.

Popkin afirma de nuevo algo interesante: "[Gassendi] Anunció entonces que estaba buscando una vía media entre pirronismo y dogmatismo. La encontró en su formulación tentativa e hipotética del atomismo epicúreo, formu-

por tanto, propia de los ingenieros y mecánicos, que fue utilizada en la construcción de artefactos; será de carácter instrumental y no ontológica.

${ }^{6}$ R. Popkin, "The religious background of the seventeenth century Philosophy", en Daniel Garber y Michael Ayers, eds., The Cambridge History of Seventeenh-Century Philosophy. Cambridge, Universidad de Cambridge, 1998, vol. I, p. 401.

${ }^{7}$ El Descartes de los Principios... también habla de certeza moral. 
lación que, en ambos aspectos, se acerca al empirismo de la moderna filosofía británica". 8

\section{La experimentación}

Como vimos en las líneas arriba expuestas, la epistemología boyleana está estrechamente ligada a la de Gassendi; además, este cofundador de la Royal Society agrega una serie de nuevos elementos del ámbito jurídico y el método experimental. Sobre lo último diremos que uno de los antecedentes importantes de Boyle, como de la mayoría de los miembros de la Royal Society, fue Francis Bacon. ${ }^{9}$ Boyle transforma lo que para Francis Bacon era el "método histórico" en un método experimental y a la filosofía natural le da el ingrediente necesario para convertirse después en una ciencia empírica. Además, es importante comentar que la experimentación fue una de las formas en las que Boyle apoyó la hipótesis mecánica.

\section{Mecanicismo}

Para comenzar con el tema cito un pasaje de Daniel Garber:

[...] en la mitad del siglo entre 1640 y 1650 surgieron tres figuras cuyos puntos de vista establecieron en cierta forma la agenda para el resto del siglo con respecto al tema [la naturaleza del cuerpo] las filosofias mecánicas de los sistemas de Gassendi, Descartes y en menor medida Hobbes definieron los temas con los que pensadores posteriores debían tratar. ${ }^{10}$

Las tres figuras arriba mencionadas, estaban preocupadas por proponer una filosofía natural alternativa a la aristotélica; la encontraron en la filosofía de Epicuro. Si bien podemos encontrar sus variantes en cada autor, como dice el pasaje arriba citado, "establecieron la agenda para el resto del siglo" sobre el tema de la naturaleza del cuerpo. Es importante decir que el impacto

${ }^{8}$ R. Popkin, op. cit., p. 162. Las cursivas son mías.

9 Como dice M. A. Stewart "Su teoría [la de Boyle] reflejaba a la nueva tradición 'mecánica' de la ciencia continental tipificada filosóficamente por Descartes y Gassendi, mientras que su método de acumulación de experimentos y observaciones debía mucho a Francis Bacon" (M. A. Stewart, ed., Selected Philosophical Papers of Robert Boyle. Hackett, Indianápolis, 1991, p. XIII).

${ }^{10}$ Daniel Garber et al., "New doctrines of body and its powers, place, and space", en The Cambridge Histoy of Seventeenh-Century Philosophy, vol. I, p. 569. 
del atomismo antiguo en la filosofía del siglo XVII se lo debemos a Gassendi, pues como dice Koyré, Gassendi le dio a la filosofía moderna una ontología: el atomismo, esta ontología, nueva para los modernos, pero antigua en Occidente, se vio como una alternativa a la filosofía aristotélica. ${ }^{11}$

Veamos ahora, cuáles son las tesis principales del mecanicismo: 1. El mecanicismo antiguo permitió a los modernos redefinir el concepto de materia, pues según la filosofía epicúrea, la materia es inerte y no puede dotarse a sí misma de ningún tipo de movimiento, organización, vida y fuerza, ya que no son propiedades de ella, pues es pasiva. En otras palabras, no posee fuerzas activas internas, nada dentro de sí misma la determina a moverse. 2. La unificación del mundo material, es decir, la sustancia material es la misma si hablamos de los cielos o de la tierra, todo está compuesto de átomos. Así, el atomismo permitió la unificación del universo, pues en el mecanicismo no hay diferencias cualitativas entre diferentes tipos de materia que no se puedan explicar en términos de movimiento y choque de partículas. ${ }^{12} 3$. El mundo, desde la perspectiva mecanicista, fue repetidamente comparado con un reloj o con un mecanismo complejo. Por ello, encontramos el argumento del designio (designio/diseño, que son las nociones que, en inglés, expresa la palabra design; en castellano debemos cuidarnos en aplicar la acepción pertinente en los casos en los que se aplique), como uno de los que más frecuentemente acompañan a las filosofías naturales del siglo XVII; en particular, para Robert Boyle representa una de las pruebas de la existencia de Dios, por tanto, una de las formas de cristianizar el atomismo antiguo. Sobre esto leeré a continuación un pasaje de Robert Boyle, que es de los más citados en la literatura sobre el tema; es el de la metáfora del Dios relojero; este pasaje aparece en $A$ Free Enquiry into the Vulgarly Received Notion of Nature, ${ }^{13}$ veamos:

${ }^{11}$ Koyré afirma que "[...] aunque [Gassendi] no contribuyó más que en muy poca medida $[\ldots]$ al desarrollo de la ciencia moderna, hizo algo mucho más importante: le aportó la ontología, o más exactamente, el complemento ontológico que le era necesario, la ontología democrítea o epicúrea [...] la que Gassendi aportó al siglo XVII y la que puso en orden de batalla contra el Estagirita" (A. Koyré, op. cit., p. 307).

${ }^{12}$ Koyré afirma algo muy interesante sobre el tema de la unificación del cielo y de la tierra que es lo siguiente: "[...] fue la concepción de Nicolás de Cusa la que inauguró el trabajo destructivo que lleva a la abolición del cosmos bien ordenado, poniendo en el mismo plano ontológico la realidad de la Tierra y la de los cielos nos dice, es una stella nobilis, una estrella noble, y es por eso mismo, tanto la afirmación de la infinitud o más bien de la indeterminación del universo, por lo que pone en funcionamiento el proceso de pensamiento que desembocará en la nueva ontología, en la geometrización del espacio y en la desaparición de la síntesis jerárquica" (A. Koyré, op. cit., p. 45).

13 Obra que lo mismo critica las posturas aristotélicas que las materialistas, pero también a la medicina de Galeno. 
[la naturaleza] es como un fino reloj, acaso como el de Estrasburgo, donde todas las cosas fueron tan hábilmente concebidas que la maquinaria, puesta una vez en movimiento, todas las cosas proceden de acuerdo con el primer designio del artífice, y los movimientos de las estatuillas, que a todas horas desempeñan éstas o aquellas cosas, no requieren, como los títeres, la peculiar interposición del artífice, o de un agente inteligente empleado por él, sino que desempeñan sus funciones en ocasiones particulares en virtud de la disposición general y primitiva de toda la maquinaria. ${ }^{14}$

En otras palabras, la adopción del mecanicismo como modelo explicativo de la naturaleza frente al dominante aristotélico, se da gracias al redescubrimiento del atomismo de Epicuro, pues éste les permitió redefinir a la materia o la naturaleza de lo corpóreo.

Boyle, al igual que la mayoría de sus contemporáneos, busca redefinir el concepto de materia frente a la noción prevaleciente propia de la filosofía aristotélica; desde el punto de vista de este autor y la mayoría de los modernos, la noción aristotélica de cuerpo apuntaba a una entidad ininteligible e inclusive volitiva. En lugar de esta perspectiva, Boyle adopta el mecanicismo y por ello, la perspectiva mecanicista de la naturaleza y así la convierte en objeto de estudio, pues gracias a la hipótesis mecánica será posible explicar los fenómenos naturales y entender la naturaleza como una máquina (como un reloj) cuyos movimientos obedecen a las operaciones mecánicas de los supuestos corpúsculos materiales; en otras palabras, la percepción mecanicista de la naturaleza permitirá a los filósofos del siglo XVII dar una explicación (causaleficiente) de los fenómenos naturales, de tal forma que éstos serán vistos como efectos del movimiento mecánico de las partículas en los cuerpos.

A este mecanicismo, cuyo antecedente es el de Epicuro, Boyle al igual que Gassendi, intentó cristianizarlo y por ello, afirma entre otras cosas, que Dios es la causa del movimiento, de la existencia, de la conservación de la materia y que ésta, una vez puesta en movimiento, Dios la guía y por esta razón, la materia se comporta de acuerdo con el orden a ella impuesto, a este orden lo llamamos leyes de la naturaleza.

Por último, el mecanicismo de Robert Boyle tanto por su énfasis en la experimentación como por sus intereses en la química en apoyo a la medicina, será una versión o una nueva ruta del mecanicismo inglés del siglo XVII; además, para no correr el riesgo de ser llamado epicúreo y por tanto ateo, Boyle prefiere ser reconocido como corpuscularista.

${ }^{14}$ R. Boyle, A Free Enquiry into the Vulgarly Received Notion of Nature, p. 13. 


\section{El mecanicismo en Inglaterra}

El atomismo y el mecanicismo que se aceptó y adoptó en la comunidad británica del siglo XVII fue el de Gassendi y no el de Descartes. La filosofía de Gassendi llegó a Inglaterra por medio de Walter Charleton (amigo de Thomas Hobbes) "quien durante un exilio voluntario en París en el periodo de la Guerra Civil" [...] conoció la filosofía natural cartesiana y la tenía en alta estima, sin embargo; “...se inclinó a favor de Gassendi en contra de Descartes” [...] La razón por la que adoptó la versión de Gassendi de la nueva filosofía presumiblemente deriva de lo que considero el conocimiento superior de Gassendi en relación con los desarrollos contemporáneos de la filosofía de la (al) química. 'De hecho es seguro afirmar que la aceptación de la filosofía de Gassendi en Inglaterra se derivó del hecho de que era más compatible con las tradiciones de la 'empiric society' de los ingleses, que la filosofía mecánica de Descartes. ${ }^{15}$

Por lo dicho en páginas anteriores, recordemos que la ruta que toma Robert Boyle en relación con el escepticismo es claramente la gassendiana. Ahora bien, sobre el mecanicismo ocurrirá en parte lo mismo, es decir, el mecanicismo de Robert Boyle es en gran medida una continuación del gassendiano, que a su vez es una actualización o puesta al día del de Epicuro.

Para apreciar el contexto en el que se desarrolla el mecanicismo de Robert Boyle, quiero expresar brevemente el contexto del mecanicismo en Inglaterra del siglo XVII.

En lo que al mecanicismo se refiere podemos hablar de tres pensadores importantes en el contexto de la filosofía natural, que son las tres figuras dominantes en este periodo sobre el mecanicismo, a saber: Descartes, Gassendi y Hobbes, como dice el pasaje de Garber arriba citado. 1. Hobbes es el pensador más importante, de un grupo muy influyente sobre el tema; conocido como el grupo de Newcastle. 2. Es probable que el mecanicismo de Robert Boyle tenga su origen como en la mayoría de los filósofos ingleses del siglo XVII, en la versión gassendiana expuesta y divulgada, además de muy influyente, de Walter Charleton. ${ }^{16}$ Con esto último, lo que quiero enfatizar es que el

${ }^{15}$ D. Garber et al., "New doctrines of Body and its powers, Place and space", en $o p$. cit., vol. I, p. 587.

${ }^{16}$ Charleton fue un gran divulgador de las ideas nuevas, en particular de la filosofía mecánica-atomista de Epicuro y Gassendi. Otro dato curioso es el de que Charleton era médico de oficio y que empezó su recorrido por la filosofía natural con Van Helmot. La obra de Charleton donde aparece la mejor presentación de la filosofía natural de 
mecanicismo de Robert Boyle es gassendiano pero no exclusiva ni directamente, pues lo conoce por medio de los trabajos de Charleton; además es una síntesis, tanto del cartesiano como del gassendiano. Como afirma Kargon:

La filosofía corpuscular de Boyle era una versión generalizada de Gassendi y el cartesianismo, muchos de sus trabajos experimentales son intentos vindicativos de las filosofías mecánicas de Descartes y Gassendi explicándolas por medio de experimentos. Más aún, al igual que Charleton, Boyle se esforzó mucho en "purificar" el atomismo y purgarlo de sus implicaciones ateas. ${ }^{17}$

Finalmente, el mecanicismo de Robert Boyle tanto por su énfasis en la experimentación como por sus intereses en la química como apoyo a la medicina, será una versión o ruta nueva dentro del mecanicismo inglés del siglo XVII.

\section{Teología: el voluntarismo teológico}

La perspectiva teológica que sostiene Boyle, que de nuevo comparte con Gassendi al igual que con Newton ${ }^{18}$ es la del voluntarismo teológico, esto significa enfatizar la libertad y el poder de Dios por encima de cualesquiera otros atributos divinos. El voluntarismo determina cierta epistemología y por ello sólo es consistente con un sistema filosófico cuya perspectiva del conocimiento sea el conocimiento probable. Igualmente, implica asumir que el mundo ha sido creado y sometido totalmente por el poder y la voluntad de Dios, cuya intervención mantiene por medio de las leyes establecidas por él. En otras palabras, el voluntarismo teológico significa tanto afirmar que Dios ha diseñado el mundo, que le haya impreso un designio; y también que nada puede restringir su poder.

Gassendi en inglés se titula Physiologia... (Cf. Robert Kargon, "Introducción", en Walter Charleton, Physiologia Epicuro-Gassendo-Charletoniana: or a Fabrik of Sciene Natural, upon the Hypothesis of Atoms, fonded by Epicurus. Introd. de Robert Kargon. Nueva York/Londres, Johnson Reprint, 1966, p. 3).

${ }^{17}$ R. Kargon, "Introducción", en op. cit., p. 11.

${ }^{18} C f$. J. W. Wojcik, "Pursuing Knowledge: Robert Boyle and Isaac Newton", en Margaret Osler, ed., Rethinking the Scientific Revolution. Cambridge, Universidad de Cambridge, 2000, pp. 184-200, donde habla de la diferente forma de relacionar el voluntarismo teológico con el conocimiento en estos dos autores; además menciona el tema de los límites del entendimiento humano como un rasgo importante de la perspectiva epistemológica de Boyle, tema que seguramente fue importante para el desarrollo de Essay... de John Locke. 
La relación entre el voluntarismo teológico y el escepticismo constructivo genera una epistemología de corte probabilista la cual, a su vez, está ligada con el empirismo como afirma Wojcik:

[...] esto es así pues desde el punto de vista del voluntarismo el conocimiento a priori es imposible, porque Dios ha escogido libremente crear este mundo en lugar de otro y la única forma de saber exactamente que tipo de mundo creó Dios es investigar el mundo en forma empírica. ${ }^{19}$

Entonces, desde ambas posiciones, tanto la teológica como la escéptica, se asume la tesis de que la estructura real y completa o total de la naturaleza es incognoscible ${ }^{20}$ sin embargo, se puede avanzar en el conocimiento de la misma, incluso ofrecer alguna evidencia de ella a través de la indagación, el juicio y el testimonio filosófico, tanto argumentativo como experimental de los hechos naturales.

\section{La alquimia}

"[la] alquimia constituye un término medio que funciona entre sus dos compromisos filosóficos potencialmente opuestos: su mundo mecánico y corpuscular y su mundo espiritual y teológico". ${ }^{21}$

A Lawrence Principe, especialista y defensor del importante papel de la alquimia en Robert Boyle, le parece sorprendente que esta relación se haya estudiado seriamente en los últimos veinte años en la obra de Newton, sin embargo, encontramos poco sobre el tema en Boyle, quien fue uno de sus antecesores más notables dentro de la arena de la filosofía natural y cofundador de la Royal Society; además de muy conocido tanto en Inglaterra como en el resto de Europa gracias a su prolífica obra, también fue un fiel, por no decir p.184

${ }^{19}$ J. W. Wojcik, "Pursuing Knowledge: Robert Boyle and Isaac Newton", en op. cit.,

20 "[Boyle] no pensaba que los seres humanos pudieran adquirir una conocimiento completo de los secretos de la naturaleza, tampoco que ningún ser humano pudiera comprender los misterios del cristianismo; pensaba que la comprensión completa era algo que Dios había reservado para la vida después de la muerte. De hecho, una comprensión completa tanto de la naturaleza como de la teología era la recompensa final de un cristiano virtuoso; este era el mejor incentivo que Dios podía ofrecer a una persona que fuera cristiana y virtuosa" (J. W. Wojcik, "Pursuing Knowledge: Robert Boyle and Isaac Newton", en op. cit., p. 197).

${ }^{21}$ Laurence Principe, "Boyle's Alchemical Pursuits", en Michael Hunter, ed., Robert Boyle Reconsidered. Cambridge, Universidad de Cambridge, 1994, p. 102. 
devoto alquimista; sin embargo, pocas son las investigaciones sobre la importancia de la alquimia para este filósofo natural.

Siguiendo las tesis de Principe, diremos en forma breve, que la alquimia no fue para Boyle un pasatiempo juvenil sino un tema importante a lo largo de su vida. Es más, según Principe: “...en los últimos años de su carrera, la alquimia por virtud de sus facetas espirituales, funciona como un enlace entre las dos esferas principales de su actividad, a saber: el avance de la filosofía natural experimental y la defensa de la fe cristiana". ${ }^{22}$

En otras palabras, no cabe la menor duda de que Boyle era un alquimista por las siguientes razones: 1. “...creía en la realidad de la trasmutación alquímica" ${ }^{23}$ 2. "...estaba convencido de la existencia de la piedra filosofal y en la validez de las afirmaciones alquímicas y de sus poderes", ${ }^{24}$ era tan firme su creencia que él mismo hizo experimentos de trasmutación de metales. De hecho, intentó elaborar su propio mercurio incandescente siguiendo las instrucciones de un texto críptico de Basilius Valentinus; también leyó los trabajos químicos y iatroquímicos de varios autores incluyendo a dos de los más notables; Van Helmot y Paracelso. 3. Nunca perdió el interés por establecer contacto con alquimistas. 4. También era un auténtico alquimista por el estilo en que escribía los reportes de sus trabajos e investigaciones como dice Principe: “...su elección cuidadosa y deliberada de las expresiones y el vocabulario creaban un alusivo sub-texto accesible sólo a lectores versados en los estilos literarios alquímicos, en los cuales se revela el objetivo original y el proceso como una preparación de la piedra filosofal". ${ }^{25}$

Esto último, nos lleva a un tema muy interesante "El secreto es un elemento crucial de la alquimia, todos los alquimistas ordenan a sus estudiantes guardar silencio, y ellos mismos, sagazmente exponían su conocimiento en un lenguaje oscuro, metafórico, en un doble mensaje que confundía y disuadía a los lectores". ${ }^{26} \mathrm{El}$ uso de un lenguaje oscuro, metafórico, que oculta al lector no versado en la alquimia puede provocar en los historiadores de la filosofía y de la ciencia, una enorme dificultad para sostener que Boyle es una de las figuras más importantes en la promoción de la comunicación abierta y la validación pública del conocimiento, en una palabra, entra en contradicción con el Boyle cofundador de una institución como la Royal Society cuyos valores principales fueron la divulgación y la validación del conocimiento, incluso con la imagen de Boyle como el padre de la química moderna, gracias, en

\footnotetext{
22 Ibid., pp. 91-92.

${ }^{23}$ Ibid., p. 93.

${ }^{24}$ Idem.

25 Ibid., pp. 93-94.

26 Ibid., p. 95.
} 
parte, al uso de la experimentación que es un método tanto de conocimiento como de comprobación del mismo.

Siguiendo las tesis de Principe me parece importante no disminuir la importancia de la alquimia en Boyle y todo lo que implica; aun cuando desde nuestra perspectiva o primera impresión, pareciera contradictorio con nuestra imagen más simple. Las investigaciones de Principe en defensa del papel de la alquimia en la obra de Robert Boyle nos permiten tener una percepción más rica, del largo y tortuoso camino que fue para los modernos establecer lo que hoy llamamos ciencia, y que en su momento la filosofía natural se encontraba entre la ciencia y el hermetismo, en otras palabras, como afirma Dascal “...las rupturas no son jamás totales, y aun si se desechan los antiguos marcos, los viejos temas no desaparecen de las telas modernas". ${ }^{27}$

Por todo ello, creemos que el revalorar la importancia de la alquimia para Boyle nos permitirá, en primer lugar, tener una interpretación más justa de los intereses y preocupaciones de este filósofo natural; incluso nos ofrecerá la posibilidad de percibir en su verdadera dimensión la realidad del pensamiento moderno, en otras palabras: entender que para que ellos pudieran romper con el aristotelismo, además de renovar filosofías antiguas, principalmente: el atomismo y el escepticismo; también incorporaron el hermetismo del Renacimiento, que pasará a la alquimia en el siglo XVII. 5. Otro rasgo que ofrece Principe como apoyo a su tesis del Boyle alquimista es el tema de los "magnetos", que según el estudioso, es un concepto propiamente alquímico.

En este tema de los magnetos, mejor conocido como el problema del magnetismo encontramos varias cuestiones interesantes, a saber: 1. En primer lugar, el problema del magnetismo relaciona a Boyle en forma muy estrecha con Newton lo cual es un apoyo importante para las tesis de Principe a favor del Boyle alquimista. 2. En segundo lugar, el tema del magnetismo es importante porque muestra los límites de la explicación mecánica, ya que la atracción magnética rebasa los alcances de este tipo de explicación. 3. Tercero, tanto por los límites explicativos del mecanicismo como por su tendencia al ateísmo, propia e inherente del atomismo, Boyle y Newton continuaron trabajando en alquimia para resolver ciertos temas que el mecanicismo no podía resolver o fatalmente provocaba.

En resumen, la importancia de la alquimia en la obra de Robert Boyle lo muestran las siguientes cuestiones: sus trabajos sobre alquimia tanto en número como en forma denotan la importancia que representaba esta área para

${ }^{27}$ Marcelo Dascal, "Lenguaje y conocimiento en la filosofía moderna”, en Ezequiel de Olaso, ed., Del Renacimiento a la Ilustración I. Enciclopedia Iberoamericana de Filosofía. Madrid, Trotta, 1994, p. 15. 
este autor; y que las razones en su caso del papel de la alquimia dentro o paralela a su filosofía natural se deben a dos cuestiones: primera, que no desarrollamos aquí, pero que para Boyle era muy importante, es su preocupación por la medicina, quizá mayor, que por la dinámica, pues Boyle estaba interesado en lo que en su época se llamaba iatroquímica que era una síntesis entre química y medicina; segunda, no por ello menos importante, es que Robert Boyle al igual que Newton pensaba que la alquimia podía contrarrestar el efecto secundario más amenazante y peligroso del atomismo: el ateísmo. ${ }^{28}$

${ }^{28}$ Como dice Principe: "Al final de su carrera Boyle percibía que la alquimia podría ayudar a construir el puente entre la filosofía experimental y la teología. La alquimia complementa y corrige el sistema de la filosofía natural que Boyle apoyaba. La alquimia era la interfase entre el mundo racional y milagroso y espiritual" (L. Principe, "Boyle's Alchemical Pursuits", en op. cit., p. 102). 
